\title{
Anti-cancer activity of glucosamine through inhibition of N-linked glycosylation
}

\author{
Viktor Chesnokov, Beata Gong, Chao Sun and Keiichi Itakura*
}

\begin{abstract}
Background: We have reported that the glucosamine suppressed the proliferation of the human prostate carcinoma cell line DU145 through inhibition of STAT3 signaling. DU145 cells autonomously express IL-6 and the IL-6/STAT3 signaling is activated. IL-6 receptor subunits are subject to $\mathrm{N}$-glycosylation, a posttranslational modification which is important for protein stability and function. We speculated that the inhibition of STAT3 phosphorylation by glucosamine might be a functional consequence of the reduced N-glycosylation of gp130.

Methods: The human prostate cancer cell lines DU145 and PC-3 and human melanoma cell line A2058 were used in this study. Glucosamine effects on N-glycosylation of glycoproteins were determined by Western blot analysis. IL-6 binding to DU145 cells was analyzed by flow cytometry. The cell proliferation suppression was investigated by colorimetric Janus green staining method.

Results: In DU145 cells glucosamine reduced the N-glycosylation of gp130, decreased IL-6 binding to cells and impaired the phosphorylation of JAK2, SHP2 and STAT3. Glucosamine acts in a very similar manner to tunicamycin, an inhibitor of protein $\mathrm{N}$-glycosylation. Glucosamine-mediated inhibition of N-glycosylation was neither protein- nor cell-specific. Sensitivity of DU145, A2058 and PC-3 cells to glucosamine-induced inhibition of N-glycosylation were well correlated to glucosamine cytotoxicity in these cells.
\end{abstract}

Conclusion: Our results suggested that the glucosamine-induced global inhibition of protein N-glycosylation might be the basic mechanism underlying its multiple biochemical and cellular effects.

Keywords: Glucosamine, N-linked glycosylation, IL-6, STAT3, Cancer

\section{Background}

In the previous paper [1], we reported that glucosamine suppressed the proliferation and induced the death of human prostate carcinoma DU145 cells. This anti-cancer activity was associated with a decreased DNA synthesis, cell cycle arrest in G1 phase, and induction of apoptosis. We demonstrated for the first time that glucosamine inhibited the phosphorylation of signal transducer and activator of transcription (STAT) 3 at Tyr705 residue, thereby inhibiting the STAT3 activity in DU145 [1]. STAT3, a member of the STAT family, is activated by phosphorylation and mediates cellular responses to cytokines and growth factors [2]. Activated STAT3 promotes angiogenesis, inflammation, proliferation and survival of cancer cells and is frequently detected in

\footnotetext{
*Correspondence: kitakura@coh.org

Department of Molecular and Cellular Biology, Beckman Research Institute of City of Hope National Medical Center, 1500 East Duarte Road, Duarte, California 91010, USA
}

numerous human tumors [3]. Suppression of the STAT3 signaling pathway [4-6] therefore represents a validated target for cancer therapy [3,7]. In DU145 cells, IL-6 is autonomously expressed and activates the IL-6/STAT3 signaling pathway by an autocrine mechanism [8]. IL-6 first binds to the two non-signaling $\alpha$-receptor subunits (IL-6R $\alpha / g p 80)$ and the resultant complex recruits the two signal transducing receptor subunits (gp130) to form the functional ternary receptor complex. Janus kinases (JAKs) associated with gp130 are activated by phosphorylation and then STAT3 becomes activated by the phosphorylated JAKs [9].

Almost all receptors on the cell membrane are Nlinked glycosylated proteins [10]. N-linked glycosylation ( $\mathrm{N}$-glycosylation) is a posttranslational modification; a preassembled core oligosaccharide moiety (glycan) is transferred to asparagine $(\mathrm{N})$ residues at potential glycosylation sites of newly synthesized polypeptides and the attached

\section{Biomed Central}


$\mathrm{N}$-linked glycans (N-glycans) are subjected to remodeling and branching [11]. N-glycosylation plays an important role for protein stability and functions $[10,12]$. Taking into account that gp130 is a highly $\mathrm{N}$-glycosylated protein [13] and that glucosamine was proposed as an inhibitor of $\mathrm{N}$-glycosylation [14], we speculated that the inhibition of STAT3 phosphorylation by glucosamine might be a functional consequence of the reduced $\mathrm{N}$-glycosylation of gp130.

In this paper, we revealed that in DU145 cells glucosamine reduced the $\mathrm{N}$-glycosylation of gp130, decreased IL-6 binding to cells and impaired the phosphorylation of JAK2 on Tyr1007/1008, SHP2 on Tyr542 and STAT3 on Tyr705 residues. Treatment with tunicamycin, an inhibitor of protein N-glycosylation [15], demonstrated all the effects of glucosamine supporting our speculation. We also showed that glucosamine-mediated inhibition of N-glycosylation was neither protein- nor cell-specific. Our results suggested that the glucosamine-induced global inhibition of protein $\mathrm{N}$-glycosylation might be the basic mechanism underlying its multiple biochemical and cellular effects.

\section{Results}

Glucosamine reduces the molecular mass of gp130 in DU145 cells by the inhibition of co-translational $\mathrm{N}$-glycosylation

To prove our hypothesis that the inhibition of STAT3 phosphorylation by glucosamine might be a functional consequence of the reduced N-glycosylation of gp130, we first studied the effect of glucosamine on the gp130 expression in DU145 cells by Western blot analysis. The treatment of DU145 cells with $2 \mathrm{mM}$ glucosamine resulted in a time-dependent accumulation of gp130 proteins with a reduced molecular mass of 100-110 kDa (marked with filled arrows) and the proteins with an original mass (marked with open arrow) became undetectable after $24 \mathrm{~h}$ (Figure 1A). However, the molecular mass of actin was unaffected. The extent of gp130 molecular mass reduction was in proportional to glucosamine concentrations (0.25-4 mM) for a $24 \mathrm{~h}$ treatment. At $0.25 \mathrm{mM}$, a slight reduction of the molecular mass was observed and at $4 \mathrm{mM}$, the original gp130 proteins completely disappeared (Figure 1B, the first row). In parallel to the reduction of the gp130 molecular mass, the phosphorylation of STAT3 at Tyr705 residue gradually decreased (the second row). It is noteworthy that the same range of glucosamine concentrations $(0.5-4 \mathrm{mM})$ in this experiment proportionally inhibited the proliferation of DU145 cells as published [1]. The observed reduction of gp130 molecular mass could occur as a result of either the loss of post-translational modifications such as $\mathrm{N}$-glycosylation, proteolytic cleavage of translated products, premature termination of translation, or a combination of these processes. Gp130 has 11 potential $\mathrm{N}$-glycosylation sites and 9 of them are actually $\mathrm{N}$-glycosylated [13]. To investigate the mechanism underlying the glucosamine-induced reduction of gp130 molecular mass, DU145 cells were treated with tunicamycin, a proven inhibitor of $\mathrm{N}$-glycosylation [15]. As shown in Figure $1 \mathrm{C}$, the tunicamycin treatment resulted in a gradual reduction of the molecular mass of gp130 in a concentration-dependent manner similar to glucosamine, albeit using about one thousandth of the concentration. The reduction of the molecular mass was accompanied by the suppression of STAT3 phosphorylation at Tyr705. These results showed that glucosamine precisely reproduced the pattern of the tunicamycin treatment (Figures 1B, C) and suggested that the glucosamine-induced reduction of gp130 molecular mass was caused by the inhibition of $\mathrm{N}$-glycosylation. To exclude the possibility of proteolytic cleavage or premature translation termination, extracts from DU145 cells cultured in the presence or absence of glucosamine were treated in vitro with $\mathrm{N}$-glycanase $\mathrm{F}$ (PNGase $\mathrm{F}$ ), which removes $\mathrm{N}$-glycans from proteins regardless of the levels of their initial $\mathrm{N}$-glycosylation (Figure 2A). Incubation of cell extracts derived from the untreated cells (lane 1, original gp130, open arrow) or the glucosamine- treated cells (lane 2, gp130 with reduced molecular mass, filled arrow) with PNGase F generated protein products with the identical molecular mass (lane 3 and 4, filled arrow). These data proved that neither proteolytic cleavage nor premature translation termination was involved in the reduction of gp130 molecular mass in the treatment with glucosamine. Furthermore, the treatment of DU145 cells with glucosamine in the presence of cycloheximide, a translational inhibitor, completely abolished the appearance of gp130 proteins with lower molecular mass (Figure 2B, lane 4). The data indicate that glucosamine does not remove $\mathrm{N}$-glycans from the mature gp130, but inhibits $\mathrm{N}$-glycosylation of the newly synthesized protein. Finally, as shown in Figure 2C, cytochalasin B, an inhibitor of glucose transporters [16], blocked the glucosamine-induced reduction of the gp130 molecular mass (lane 4) suggesting that cells uptake glucosamine by glucose transporters. Altogether, these results clearly demonstrate that glucosamine is transported into DU145 cells via glucose transporters and suppresses co-translational N-glycosylation to produce gp130 proteins with a lower molecular mass.

\section{Glucosamine-induced inhibition of N-glycosylation of gp130 represses the IL6/JAK/STAT3 signaling in DU145 cells}

To determine whether the deficiency in N-glycosylation has any effects on the activity of the gp130-associated IL-6/JAK/STAT3 signaling [9], we carried out the following investigations. First, we studied IL-6 binding to DU145 cells in the presence and absence of glucosamine. Cells were pre-treated with glucosamine (2 $\mathrm{mM}$ for $24 \mathrm{~h}$ ) and IL-6 binding to the cells were analyzed. The flow 


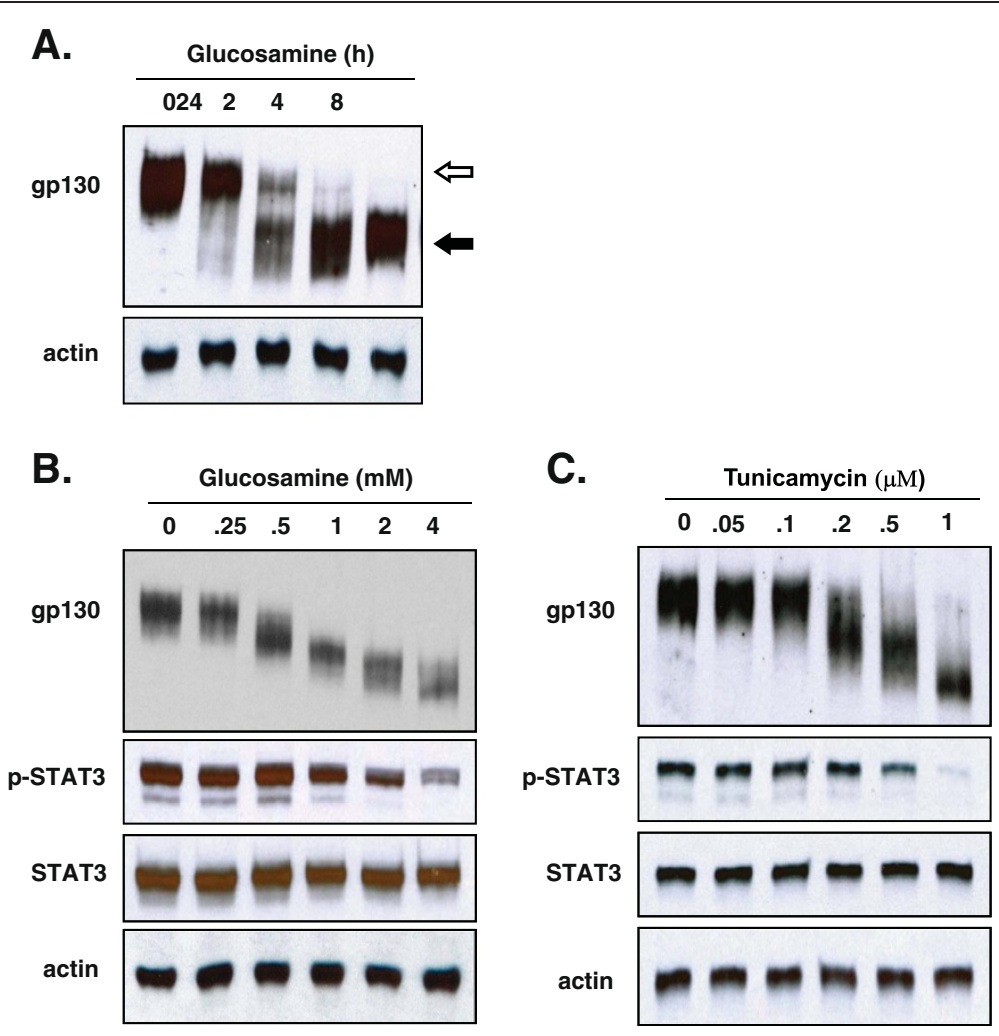

Figure 1 Glucosamine reduced the molecular mass of gp130 and suppressed the phosphorylation of STAT3 in DU145 cells. (A) Western blot analysis of the extracts from the cells cultured in the presence of $2 \mathrm{mM}$ glucosamine for the indicated time (h). Whole-cell lysates were subjected to immunoblotting using antibodies specific for gp130 and actin (loading control). The open arrow indicates the molecular mass of gp130 without glucosamine treatment and the filled arrow indicates the reduced molecular mass of gp130 following the treatment. (B) Western blot analysis of cells cultured with indicated concentrations of glucosamine $(\mathrm{mM})$ for $24 \mathrm{~h}$. Whole-cell lysates were subjected to immunoblotting using antibodies specific for gp130, phospho (Tyr705)-STAT3 (p-STAT3), STAT3 and actin (loading control). (C) Western blot analysis of cells cultured with indicated concentrations of tunicamycin $(\mu \mathrm{M})$ for $24 \mathrm{~h}$. Whole-cell lysates were subjected to immunoblotting using the same antibodies as described for $\mathbf{B}$. Each blot is a representative of three independent experiments.

cytometry binding assays revealed that the preincubation of DU145 cells with glucosamine considerably shifted the intensity of IL-6 fluorescence to a lower side indicating less binding of IL-6 to cells as compared to the untreated control (Figure 3A). Next, we analyzed the tyrosine phosphorylation of the down-stream signaling molecules of IL-6 receptor including JAK2, STAT3 and SHP2. DU145 cells secrete IL-6, which stimulates the phosphorylation of these molecules via an autocrine fashion [8]. As shown in Figure 3B, basal levels of the phosphorylated JAK2 (Tyr1007/1008, p-JAK2), STAT3 (Tyr705, p-STAT3) and SHP2 (Tyr542, p-SHP2) were detected (lane 1), and exogenous IL-6 (2 $\mathrm{ng} / \mathrm{ml}, 15 \mathrm{~min})$ further increased the tyrosine phosphorylation of these signaling proteins (lane 2). Glucosamine treatment decreased the levels of both the basal (lane 1 vs. 3) and IL-6-induced (lane 2 vs. 4) tyrosine phosphorylation of JAK2, STAT3 and SHP2. These results demonstrated that glucosamine prevented IL-6 from binding to cells and suppressed the multiple steps of the IL-6/JAK/STAT3 signaling pathway. To confirm further that the inhibition of N-glycosylation could be responsible for the decrease of IL-6 binding to cells and the impairing of the IL-6/JAK/STAT3 signaling, DU145 cells were treated with tunicamycin in a similar way to glucosamine. The results showed that tunicamycin reduced IL-6 binding to cells (Figure 3C) and impaired both the basal and IL-6-induced tyrosine phosphorylation of JAK2, STAT3 and SHP2 (Figure 3D, lane 3 and 4). We concluded that the glucosamine-induced inhibition of gp130 N-glycosylation reduced IL-6 binding to cells to suppress the IL-6/JAK/STAT3 signaling pathway.

\section{Glucosamine affects on multiple $\mathrm{N}$-glycosylated proteins}

To demonstrate that the glucosamine-induced inhibition of $\mathrm{N}$-glycosylation was not limited to gp130, we analyzed the effect of glucosamine on the other $\mathrm{N}$-glycosylated protein EGFR in DU145 cells. The $170 \mathrm{kDa}$ human EGFR has 11 potential N-glycosylation sites, 8 of them are fully glycosylated, and one site is only partially glycosylated [17]. As shown in the upper panel of Figure 4A, treatment of cells with glucosamine $(2 \mathrm{mM})$ resulted in the reduction of the expression level of the $170 \mathrm{kDa}$ EGFR protein 

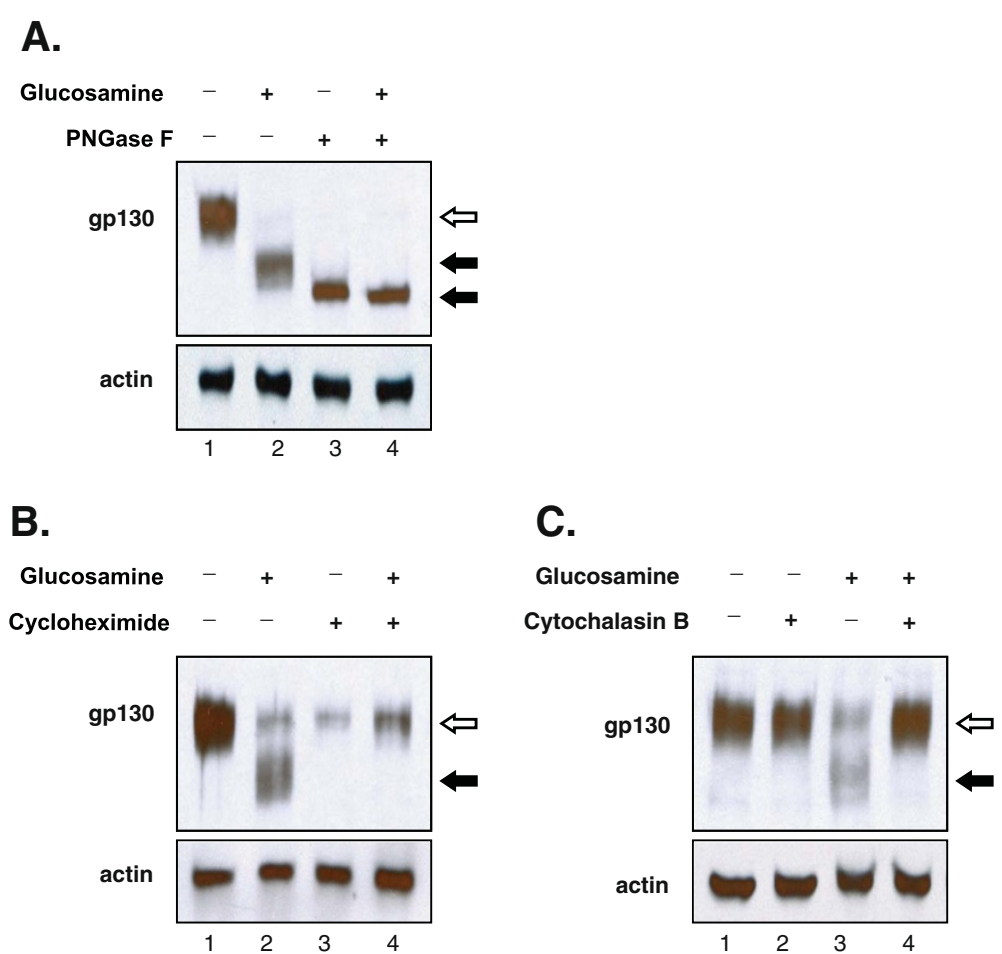

Figure 2 Glucosamine inhibited co-translational N-glycosylation of gp130 and glucose transporter activity was essential for the inhibition. (A) Western blot analysis of the whole-cell lysates treated in vitro with peptide-N-glycosidase F (PNGase F). DU145 cells cultured with or without 2 mM glucosamine for $24 \mathrm{~h}$, and then whole-cell lysates were prepared and treated with or without peptide-N-glycosidase $\mathrm{F}(40 \mathrm{ug} / \mathrm{ml})$ for $4 \mathrm{~h}$ at $37 \mathrm{C}$ followed by immunoblotting using antibodies specific for gp130 and actin (loading control). The open arrow indicates the molecular mass of N-glycosylated gp130 without glucosamine or PNGase F treatment and the filled arrow indicates reduced molecular mass of N-glycosylation deficient gp130. (B) Western blot analysis of cells treated with $2 \mathrm{mM}$ glucosamine in the presence or absence of cycloheximide. DU145 cells cultured with or without $2 \mathrm{mM}$ glucosamine for $4 \mathrm{~h}$ in the presence or absence of cycloheximide $(100 \mathrm{\mu g} / \mathrm{ml})$, and then the whole-cell extracts were prepared and subjected to immunoblotting using antibodies specific for gp130 and actin (loading control). The open arrow indicates the molecular mass of N-glycosylated gp130 and the filled arrow indicates the reduced molecular mass of N-glycosylation deficient gp130. (C) Western blot analysis of DU145 cells treated with glucosamine in the presence or absence of glucose transporter inhibitor cytochalasin B. Cells pre-incubated with $10 \mu \mathrm{M}$ cytochalasin B for $30 \mathrm{~min}$ and then treated with $2 \mathrm{mM}$ glucosamine for $4 \mathrm{~h}$. The whole-cell extracts were prepared and subjected to immunoblotting using antibodies specific for gp130 and actin (loading control). The open arrow indicates the molecular mass of $\mathrm{N}$-glycosylated gp130 and the filled arrow indicates the reduced molecular mass of N-glycosylation deficient gp130. Each blot is a representative of three independent experiments.

(marked with open arrows) and the appearance of proteins with a lower molecular mass (marked with filled arrow) in a time dependent manner as it was observed for gp130 (Figure 1A). We verified that neither proteolytic cleavage nor premature translation termination caused the reduction of the molecular mass by glucosamine (Figure 4B). Incubation of extracts from both the untreated (lane 1) and glucosamine treated cells (lane 2) with PNGase F gave de-N-glycosylated EGFR proteins with an identical molecular mass (lanes 3 and 4). Since the effect on EGFR was identical to that on gp130 (Figures 1A and 2A), we concluded that glucosamine suppresses N-glycosylation of EGFR. EGFR is a tyrosine kinase receptor which is activated by autophosphorylation at Tyr845 residue upon ligand binding [18] and we examined whether the activity was altered by de-N-glycosylation. As shown on Figure 4A, the non-treated $170 \mathrm{kDa}$ form of EGFR was strongly phosphorylated at Tyr 845 residue in the cells cultured with $10 \%$ serum (lane 1 marked with open arrow), whereas EGFR with a lower molecular mass that was deficient in $\mathrm{N}$-glycosylation was weakly phosphorylated at this residue (lanes 2 and 3 marked with filled arrow). To further confirm that glucosamine inhibited global protein N-glycosylation, in addition to gp130 and EGFR, we analyzed four other $\mathrm{N}$-glycosylated proteins including C-MET, clusterin, CD44 and MRP1 in DU145 cells. Western blot analysis of cell extracts revealed the time dependent increase of the mobility of those proteins in the presence of glucosamine (Figure 5A). Taken all the results together, we concluded that in DU145 cells glucosamine depleted a pool of $\mathrm{N}$-glycosylated proteins by suppressing $\mathrm{N}$-glycosylation of the newly synthesized proteins. To address the functional consequence of the glucosamine-induced inhibition of global protein 
A.

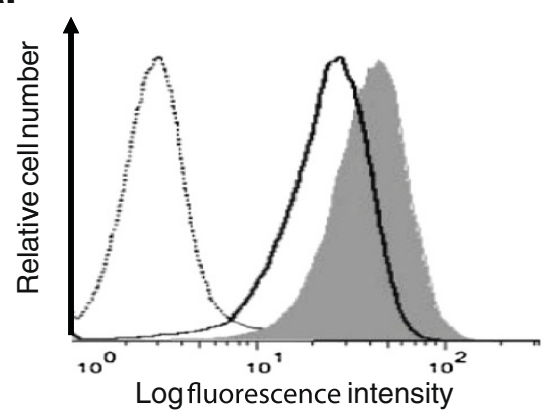

B.

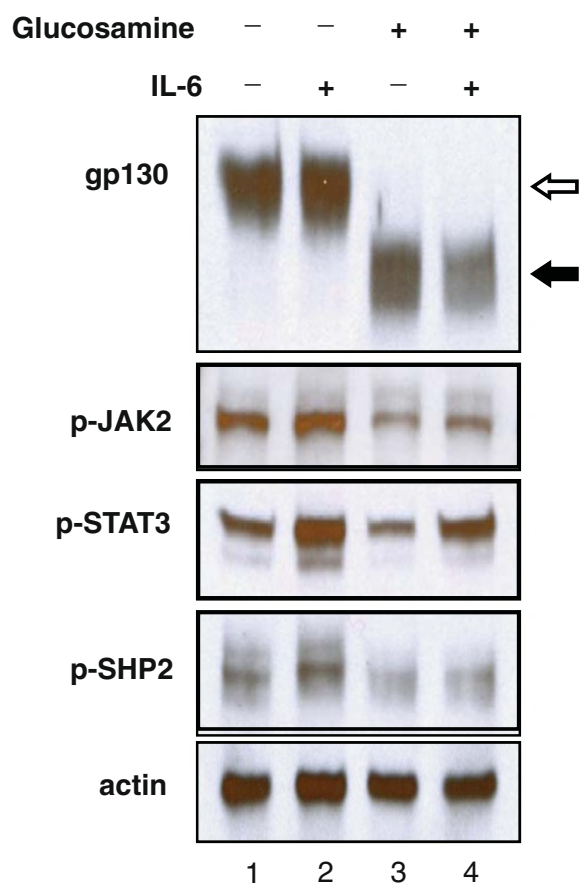

C.

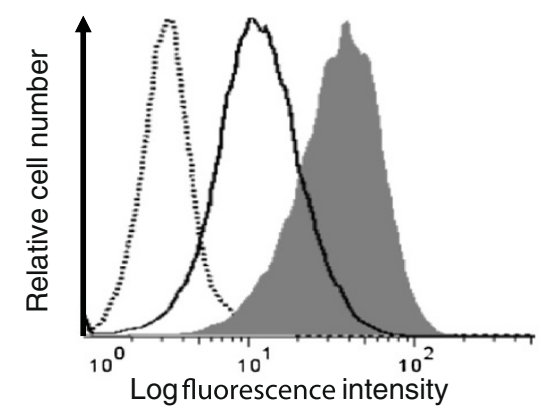

D.
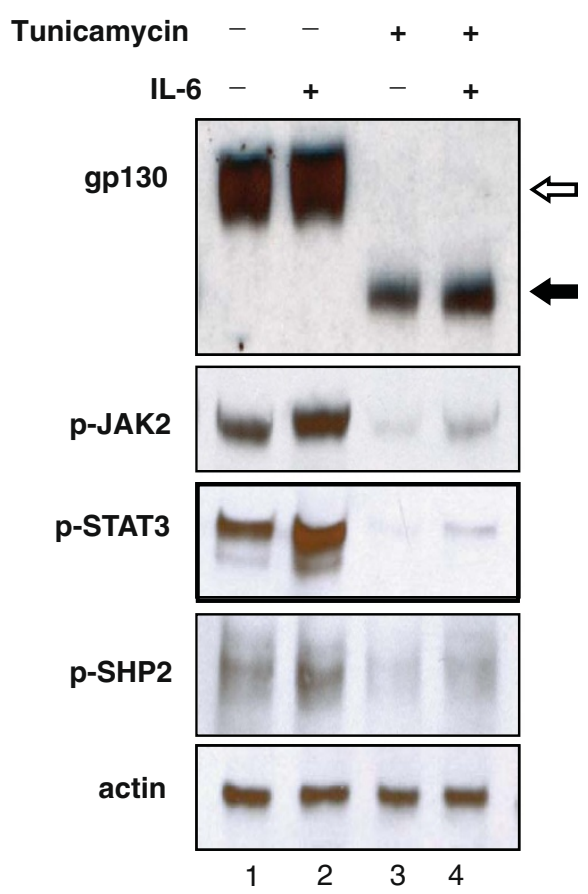

Figure 3 Glucosamine suppressed the IL-6/JAK/STAT3 signaling pathway in DU145 cells. (A) Glucosamine decreased IL-6 binding to DU145 cells. Cells cultured with or without $2 \mathrm{mM}$ glucosamine for $24 \mathrm{~h}$, and then incubated with a fluorescently labeled IL-6 followed by FACS analysis. Gray histogram represents IL-6 binding to cells without glucosamine treatment, bold line histogram represents IL- 6 binding to glucosamine-treated cells and dotted line histogram represents negative control, cells without fluorescently labeled IL-6. (B) Western blot analysis of cells cultured with or without glucosamine following the IL-6 treatment. Cells cultured in serum free medium with or without $2 \mathrm{mM}$ glucosamine for $24 \mathrm{~h}$ and then treated with $2 \mathrm{ng} / \mathrm{ml} \mathrm{IL-6}$ for $15 \mathrm{~min}$. Whole-cell lysates were subjected to immunoblotting using antibodies specific for gp 130, phospho (Tyr1007/ 1008)-JAK2 (p-JAK2), phospho (Tyr705)-STAT3 (p-STAT3), phospho (Tyr542)-SHP2 (p-SHP2) and actin (loading control). The open arrow indicates $\mathrm{N}$-glycosylated gp130 and the filled arrow indicates N-glycosylation deficient gp130. (C) Tunicamycin decreases IL-6 binding to DU145 cells. Cells were cultured with or without $1 \mu \mathrm{g} / \mathrm{ml}$ tunicamycin for $24 \mathrm{~h}$ and then incubated with a fluorescently labeled IL-6 following FACS analysis. Gray histogram represents IL-6 binding to cells without tunicamycin treatment, bold line histogram represents IL-6 binding to tunicamycin-treated cells and dotted line histogram represents negative control, cells without fluorescently labeled IL-6. (D) Western blot analysis of cells cultured with or without tunicamycin followed by the IL-6 treatment. Cells cultured in serum free medium with or without $1 \mu \mathrm{g} / \mathrm{ml}$ tunicamycin for $24 \mathrm{~h}$ and then treated with $2 \mathrm{ng} / \mathrm{ml} \mathrm{IL-6}$ for $15 \mathrm{~min}$. Whole-cell lysates were analyzed and data were presented in the same way as for glucosamine (Figure 3B). The binding assays and each blot are a representative of three independent experiments.

N-glycosylation, we examined the phosphorylation (activity) of three important signaling proteins in cancer cells, STAT3 (Tyr705), AKT (Ser473) and ERK1/2 (Thr202/Tyr204) that were activated by membrane $\mathrm{N}$-glycosylated receptors. We found that phosphorylation of all these proteins in cells cultured with serum (10\% FBS) was suppressed by the treatment of $2 \mathrm{mM}$ glucosamine in a time dependent manner (Figure 5B, lanes 2 and 3). These data suggest that suppression of multiple signaling pathways may contribute to the glucosamine anti-cancer activity. 


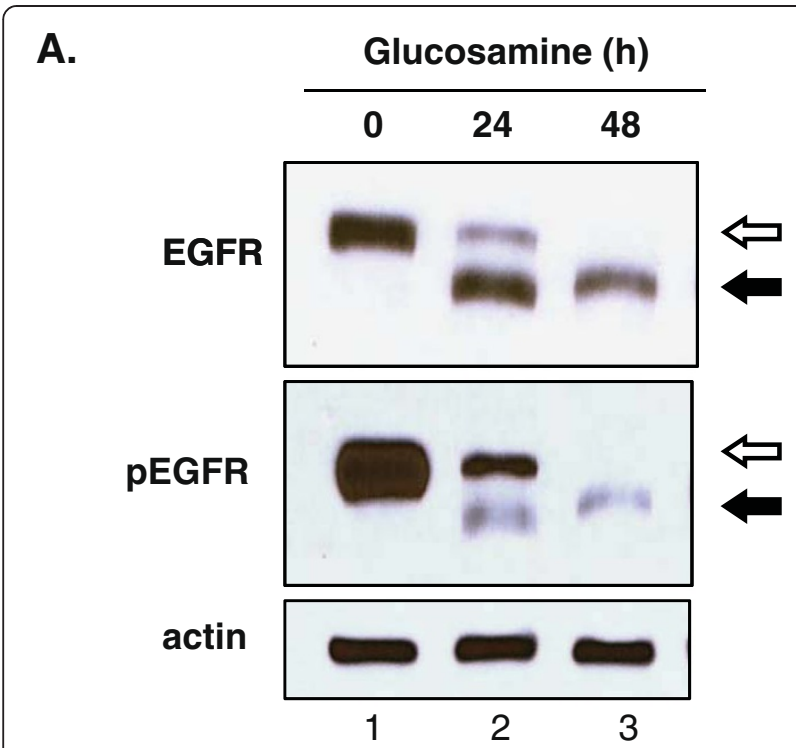

B.
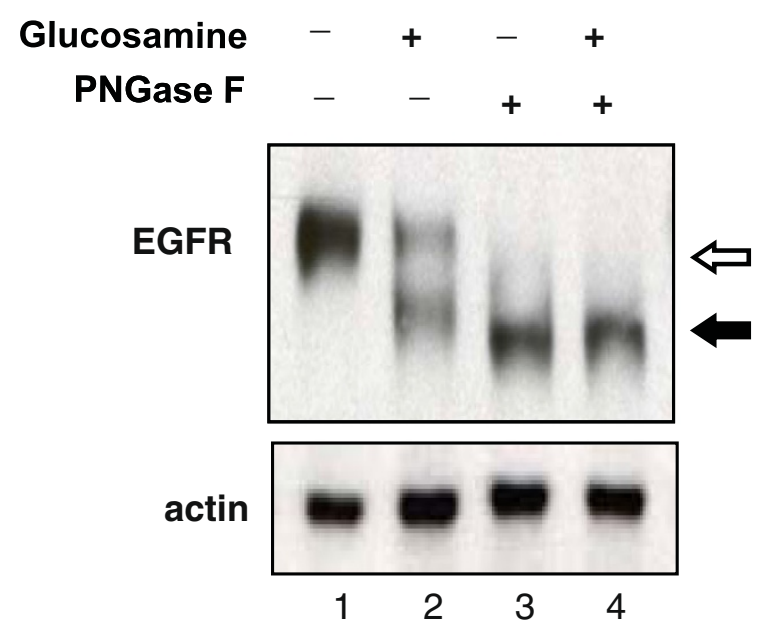

Figure 4 Glucosamine inhibited N-glycosylation and autophosphorylation of EGFR in DU145 cells. (A) Western blot analysis of cells cultured in the presence of $2 \mathrm{mM}$ glucosamine for 24 or $48 \mathrm{~h}$. Whole-cell lysates were subjected to immunoblotting using antibodies specific for EGFR, phospho (Tyr845)-EGFR (pEGFR) and actin (loading control). The open arrow indicates the N-glycosylated receptor and its phosphorylated form, and the filled arrow indicates the N-glycosylation deficient receptor and its phosphorylated form. (B) Western blot analysis of the whole-cell lysates treated in vitro with peptide-Nglycosidase F (PNGase F). DU145 cells cultured with or without $2 \mathrm{mM}$ glucosamine for $24 \mathrm{~h}$, and then the whole-cell lysates were prepared and treated with or without peptide-N-glycosidase $\mathrm{F}(40 \mathrm{ug} / \mathrm{ml})$ for $4 \mathrm{~h}$ at $37 \mathrm{C}$ followed by immunoblotting using antibodies specific for EGFR and actin (loading control). The open arrow indicates the molecular mass of N-glycosylated EGFR and the filled arrow indicates the reduced molecular mass of N-glycosylation deficient EGFR. Each blot is a representative of three independent experiments.
Sensitivity of cells to glucosamine-induced inhibition of $\mathrm{N}$-glycosylation is correlated to glucosamine anti-cancer activity

To examine whether the inhibition of $\mathrm{N}$-glycosylation is cell-specific, we compared the sensitivity of two human prostate carcinoma DU145 and PC-3 cells and human melanoma A2058 cells to glucosamine. The cells were cultured in $0.5,1,2$, or $4 \mathrm{mM}$ glucosamine for $4 \mathrm{~h}$, and the inhibition of $\mathrm{N}$-glycosylation was assessed by Western blot analysis of clusterin. As shown in Figure 6A, an increased mobility of clusterin with the inhibition of $\mathrm{N}$ glycosylation was detected at $1 \mathrm{mM}, 2 \mathrm{mM}$ and $4 \mathrm{mM}$ in DU145 cells, A2058 cells and PC-3 cells, respectively. Western blot analysis of the others $\mathrm{N}$-glycosylated proteins (gp130 and EGFR) in the respective cells produced the results identical to clusterin (data not shown). These data indicate that DU145 cells were the most sensitive to the glucosamine-induced inhibition of $\mathrm{N}$-glycosylation, followed by A2058 cells, and PC-3 cells were the least sensitive. To investigate whether the inhibition of $\mathrm{N}$ glycosylation correlated to the anti-cancer activity of glucosamine, we carried out cell viability assays of these cells under the same conditions. As shown in Figure 6B, the toxicity of glucosamine to DU145, A2058 and PC-3 cells correlated directly with the inhibition of $\mathrm{N}$-glycosylation. These results suggest that the glucosamineinduced inhibition of protein $\mathrm{N}$-glycosylation might be one of the basic mechanisms that accounts for the glucosamine anti-cancer activity.

\section{Discussion}

Glucosamine, an amino monosaccharide has been widely used as a dietary supplement to relieve discomfort of rheumatoid arthritis or osteoarthritis for more than fifty years [19]. Glucosamine has also been reported as an inhibitor of tumor growth both in vivo [20] and in vitro [21]. However, the mechanism for the anti-cancer activity is still not fully understood to explore the potentials of glucosamine as an anti-cancer agent. Previously [1], we have proposed our hypothesis that glucosamine could inhibit the N-glycosylation of IL-6 receptor in human prostate carcinoma DU145 cells, thereby reducing the activity of the IL-6/JAK/STAT3 signaling pathway. This pathway is activated and contributes to carcinogenesis in many different tumors. This study focused on proving our hypothesis and examining the mechanism by which the STAT3 activity is suppressed by glucosamine. When DU145 cells were treated with glucosamine, Western blot analysis showed that several gp130 proteins with a lower molecular mass appeared in a time and dose dependent manner (Figure 1). In the following experiments, we demonstrated that digestion of the extracts from the untreated and glucosamine-treated DU145 cells with peptide-Nglycosidase F (PNGase F), which removed all N-linked 


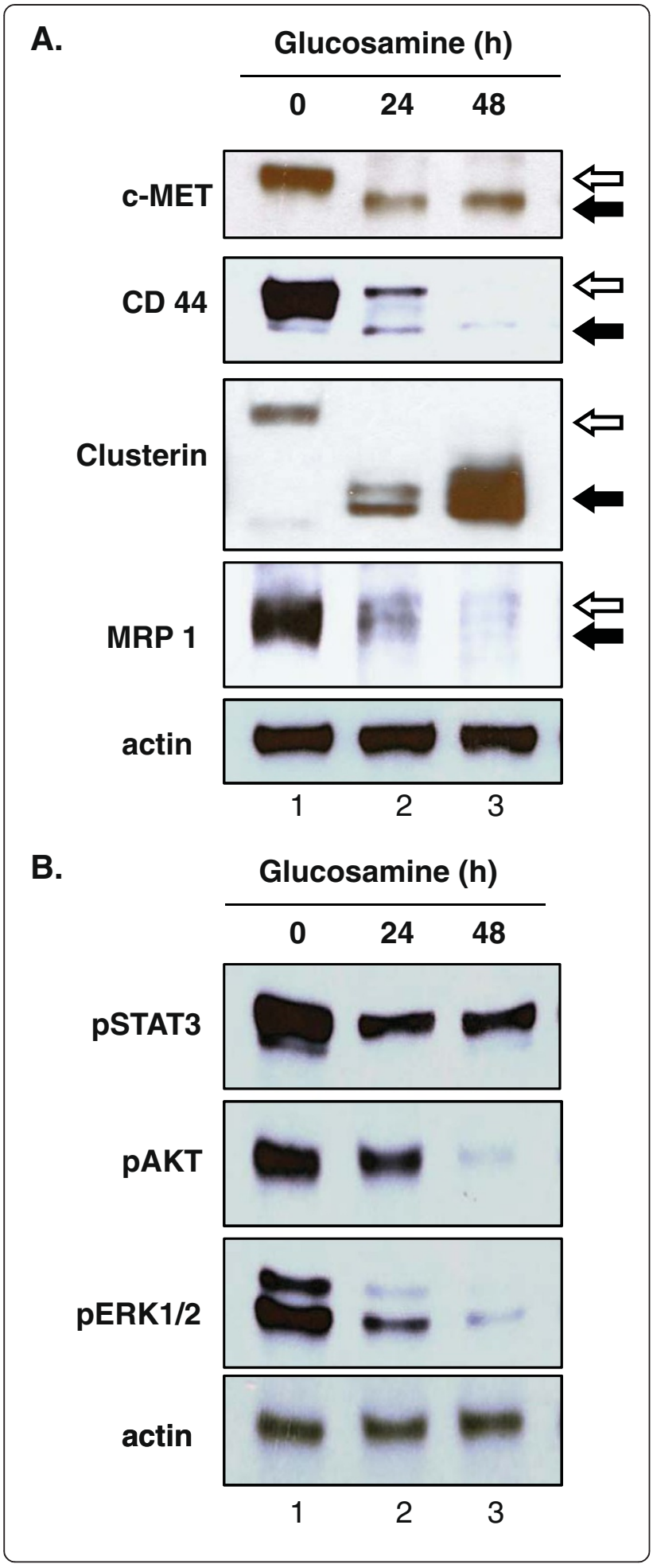

Figure 5 Glucosamine reduced the molecular mass of various glycoproteins by inhibiting $\mathrm{N}$-glycosylation and suppressed the multiple signaling pathways in DU145 cells. (A) Western blot analysis of cells cultured in the presence of $2 \mathrm{mM}$ glucosamine for 24 or $48 \mathrm{~h}$. Whole-cell lysates were prepared and subjected to immunoblotting using antibodies specific for c-MET, CD 44, clusterin, MRP1 and actin (loading control). The open arrow indicates the molecular mass of proteins without glucosamine treatment and the filled arrow indicates the reduced molecular mass of protein following the treatment. (B) Western blot analysis of the phosphorylated (active) STAT, AKT and ERK1/2 proteins after glucosamine treatment. The wholecell lysates employed in Figure 5A were subjected to immunoblotting using antibodies specific for phospho (Tyr705)-STAT3 (pSTAT3), phospho (Ser473)-AKT (pAKT), phospho (Thr202/Tyr204)-ERK1/2 (pERK1/2) and actin (loading control). Each blot is a representative of three independent experiments.

glycans [22], provided proteins with the same mobility on Western blot gels regardless of the mobility of the proteins before the digestion. These results indicated that gp130 proteins with a lower molecular mass had less $\mathrm{N}$-glycosylated sites and therefore, were not truncated products of translation step. Furthermore, the glucosamine treatment of DU145 cells in the presence of cycloheximide did not produce any gp130 proteins with a lower molecular mass suggesting that glucosamine inhibited $\mathrm{N}$-glycosylation of the newly synthesized protein but did not remove $\mathrm{N}$-glycans from the mature gp130 (Figure 2). IL-6 receptor is composed of four subunits, two gp80 subunits containing the IL- 6 binding domain and two gp130 subunits containing the signal transducing domain. Flow cytometry binding assays showed that IL-6 bound weakly to cells treated with glucosamine. We speculated that the gp130 subunit deficient in $\mathrm{N}$-glycosylation could not form the intact IL-6 receptor with the gp80 subunit and thereby the binding of IL-6 to cells was suppressed to reduce the signal transduction. It was shown that N-glycosylationdeficient gp130 after tunicamycin treatment lost the ability to form a heterodimer with the leukemia inhibitory factor (LIF) binding subunit, and lost the signal transduction in response to LIF in neuroepithelial cells [23]. To further confirm that glucosamine acts in a similar manner to tunicamycin, we carried out the same experiments described above using tunicamycin in place of glucosamine. Tunicamycin is a proven inhibitor of protein $\mathrm{N}$-glycosylation which blocks the transfer of $\mathrm{N}$-acetylglucosamine to dolichol in the first step of oligosaccharide synthesis [15]. Indeed, tunicamycin lowered the molecular mass of the normally expressed gp130, suppressed IL-6 binding to cells and reduced the activity of the IL-6/JAK/ STAT3 signaling pathway in a dose dependent manner like glucosamine (Figure 1C, 3). Obviously, various proteins are $\mathrm{N}$-glycosylated and the modification of proteins by $\mathrm{N}$-glycosylation is not specific to gp130. To confirm that these $\mathrm{N}$-glycosylated proteins are substrates for 


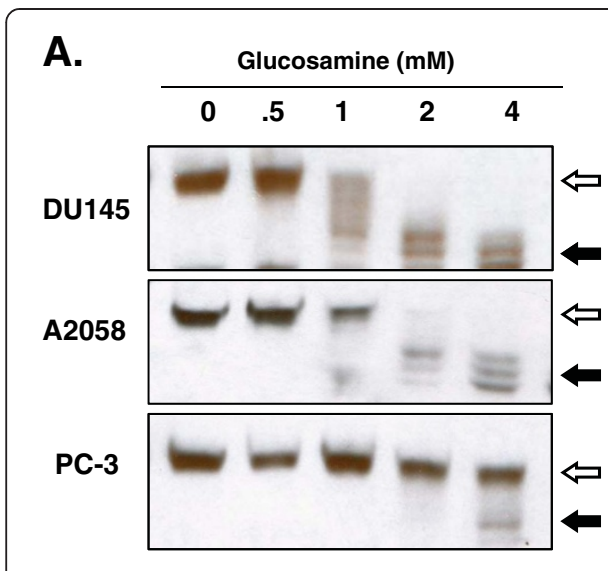

B.

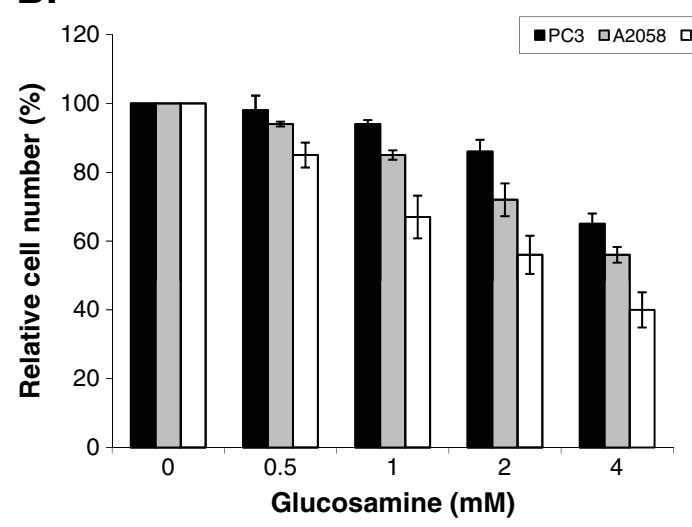

Figure 6 The sensitivity of cells to the inhibition of Nglycosylation by glucosamine was correlated to glucosamine toxicity. (A) Western blot analysis of DU145, A2058 and PC-3 cells cultured with the indicated concentrations of glucosamine (mM) for $4 \mathrm{~h}$. Whole-cell lysates were subjected to immunoblotting using antibodies specific for clusterin as N-glycosylation marker. The open arrow indicates the molecular mass of $\mathrm{N}$-glycosylated clusterin and the filled arrow indicates $\mathrm{N}$-glycosylation deficient clusterin. Each blot is a representative of three independent experiments. (B) The viability of DU145, A2058 and PC-3 cells cultured in the presence of the indicated concentration ( $\mathrm{mM}$ ) of glucosamine for $48 \mathrm{~h}$. The viability of cells was measured by cell counting using the colorimetric Janus green whole-cell stain method. The results were present as mean+/-SD of four independent experiments.

glucosamine, we carried out the Western blot analysis of several glycoproteins, EGFR, c-MET, CD44, clusterin and MRP1 after the glucosamine treatment. In addition to gp130, the treatment produced these glycoproteins with a lower molecular mass in a different extent (Figure 5A). Since most of cell-surface receptors are $\mathrm{N}$-glycosylated [10], it was expected that multiple signaling pathways activated from these receptors were affected by glucosamine. Indeed, Western blot analysis showed that in addition to STAT3, the phosphorylation (activity) of other proteins playing key signaling roles in cancer cells, AKT and ERK1/2, were suppressed after the treatment with glucosamine in DU145 cells (Figure 5B). These results suggest that glucosamine-induced inhibition of global protein $\mathrm{N}$-glycosylation might be the basic mechanism of multiple biochemical and cellular events of its anticancer action. In fact, the sensitivity of different cells to glucosamine measured by de- $\mathrm{N}$-glycosylation of clusterin was in a good agreement with their viability (Figure 6). As it was proposed, targeting multiple signaling pathways by disrupting posttranslational N-glycosylation in a combination with other agents might be an effective strategy for cancer therapy [24]. For example, tunicamycin treatment enhanced the susceptibility of human nonsmall cell lung cancer cells to EGFR tyrosine kinase inhibitor erlotinib [25], and also stimulated TRAILinduced apoptosis in human prostate cancer PC-3 cells [26]. Unfortunately, tunicamycin is too toxic to use for the human treatment [27]. Alternatively, glucosamine could be a candidate for clinical use as an inhibitor of posttranslational $\mathrm{N}$-glycosylation because of its low toxicity to the normal cells and enhanced uptaken by tumor cells due to "Warburg effect" [28]. We will investigate this possibility and study the mechanism underlying inhibition of $\mathrm{N}$-glycosylation by glucosamine.

\section{Conclusions}

Current study found that, in human prostate carcinoma cell line DU145, glucosamine reduced N-glycosylation of IL-6 receptor subunit gp130 by inhibiting co-translational $\mathrm{N}$-glycosylation, resulting in less IL-6 binding to cells and less phosphorylation (activation) of down-stream JAK2 and STAT3 proteins. Our results demonstrated that glucosamine-mediated inhibition of $\mathrm{N}$-glycosylation was neither protein- nor cell-specific. Glucosamine induced global inhibition of protein $\mathrm{N}$-glycosylation in all three cancer cell lines which were used in this study. Several important signals, such as STAT3, AKT and ERK1/2, were activated by $\mathrm{N}$-glycosylated membrane receptors. The treatment of glucosamine led to the suppression of these signals, suggesting that the glucosamine-induced inhibition of $\mathrm{N}$-glycosylation might be the basic mechanism underlying its biochemical and cellular effects.

\section{Methods}

Cell culture, chemical compounds and biological reagents The human prostate cancer DU145 and PC3 cells and human melanoma A2058 cells were obtained from the American Type Culture Collection. Cells were cultured in RPMI medium 1640 supplemented with glutamine, essential amino acids, $10 \%$ fetal bovine serum and antibiotics (100 units/ml penicillin $\mathrm{G}$ and $100 \mathrm{ug} / \mathrm{ml}$ streptomycin sulfates). Cells were incubated at $37^{\circ} \mathrm{C}$ in $5 \% \mathrm{CO} 2$, and the medium was changed every 3-4 days. Cells were passaged at $70 \%$ confluent using trypsin/EDTA. Treatments of cells with D-glucosamine hydrochloride, tunicamycin, cycloheximide, cytochalasin B and interleukin-6 (IL-6) were 
performed in 6-well plates (Corning, Inc., Corning, NY). D-glucosamine hydrochloride, tunicamycin, cycloheximide, propidium iodide and cytochalasin B were purchased from Sigma Chemical Co., (St. Louis, MO; G-1514, T-7765, C-4859, C-6762, P-1607, and C2743). IL-6 and Human IL-6 Flow cytometry Kit were purchased from R \& D Systems (Minneapolis, MN; 206-IL, NF600). Peptide: Nglycanase F (PNGase F) was purchased from New England Biolabs (Ipswich, MA; PO704). Antibodies used for immunoblot (Western) analysis included anti-gp130, anti-clusterin (Millipore, Danvers, MA; 06-291, 05-354), anti-STAT3, anti-phospho (Tyr705)-STAT3, anti-phospho (Tyr1007/1008)-JAK2, anti-phospho (Tyr542)-SHP2, anti-EGFR, anti-phospho (Tyr845)-EGFR, anti-phospho (Ser473)-AKT, anti-phospho (Thr202/Tyr204)-ERK1/2, anti-c-Met, anti-CD44, anti-mouse IgG HRP-linked and anti-rat IgG HRP-linked from Cell Signaling Technology (Beverly, MA; 9132, 9145, 3771, 3751, 2232, 2231, 4060, 4370, 3127, 5640, 7076, 7077), anti-actin and anti-MRP1 from Santa Cruz Biotechnology (Santa Cruz, CA; 1615, 59607). Additionally, Janus Green whole-cell stain was purchased from ThermoScientific-Pierce (Rockford, IL; 62203).

\section{Western blotting}

After removing the culture medium cells in 6-well plates were washed with $1 \times$ PBS and then lysed in the wells with $0.1 \mathrm{ml}$ of RIPA lysis and extraction buffer $(25 \mathrm{mM}$ Tris- $\mathrm{HCl}, 150 \mathrm{mM} \mathrm{NaCl}, 1 \% \mathrm{NP}-40,1 \%$ Sodium deoxycholate, 0.1\% SDS, pH 7.6, (G-Bioscience, St. Louis, MO; 786-489,) supplemented with protease and phosphatase inhibitor cocktail (ThermoScientific-Pierce, Rockford, IL; 78440) for $15 \mathrm{~min}$ at $4^{\circ} \mathrm{C}$. Lysates were then transferred to $1.5 \mathrm{ml}$ microcentrifuge tubes, vortexed at maximum speed for $15 \mathrm{sec}$ to shear DNA and centrifuged at $12000 \mathrm{~g}$ for $10 \mathrm{~min}$ at $4^{\circ} \mathrm{C}$. Supernatants were quantified for protein concentrations by BCA protein assay kit (ThermoScientific-Pierce, Rockford, IL; 23227) and stored at $-80^{\circ} \mathrm{C}$ in aliquots or used immediately for SDS-PAGE. The samples for SDS-PAGE were prepared by incubating with $4 \% \mathrm{SDS}$ at $37^{\circ} \mathrm{C}$ for $30 \mathrm{~min}$ for solubilizing $\mathrm{N}$ glycosylation deficient proteins and then boiling in the sample buffer (50 mM Tris-HCI pH 6.8, 2\% SDS, 10\% glycerol, 1\% $\beta$-mercaptoethanol, 12.5 mM EDTA, 0.02\% bromophenol blue) for $5 \mathrm{~min}$. Equal amounts of proteins were then loaded onto $8 \%$ precast SDS-PAGE gels (ThermoScientific-Pierce, Rockford, IL; 25200). Immunoblotting was performed after the electrophoretic transfer of proteins onto nitrocellulose membrane (Bio-Rad Laboratories, Hercules, CA; 162-0115). The proteins were detected using protein-specific first antibodies, horseradish peroxidase-conjugated second antibodies and chemiluminescent detection reagent (Denville Scientific, Metuchen, NJ; E2500) according to the manufacturer's conditions. Protein sizes on nitrocellulose membranes were detected by protein markers (Bio-Rad Laboratories, Hercules, CA; 161-0374). To reuse, nitrocellulose membranes were washed with western blot stripping buffer (ThermoScientific-Pierce, Rockford, IL; 46430) and blocked again.

\section{Peptide: N-glycanase F (PNGase F) treatment}

To remove $\mathrm{N}$-glycans from glycoproteins in vitro aliquots of whole cell extracts in RIPA buffer were incubated with Peptide:N-glycanase F $(40 \mu \mathrm{g} / \mu \mathrm{l})$ at $37^{\circ} \mathrm{C}$ for $4 \mathrm{~h}$ and then stored at $-80^{\circ} \mathrm{C}$ or used immediately for Western blot analysis.

\section{Evaluation of IL- 6 binding to DU145 cells by flow cytometry} The Human IL-6 Flow Cytometry Kit (R \& D Systems) was utilized for analysis of IL- 6 binding according to the manufacturer's protocol with slight modifications. Briefly, DU145 cells were harvested by the treatment with $1 \mathrm{mM}$ EDTA for 3-5 min, washed two times with phosphatebuffered saline (PBS), resuspended in PBS $\left(4 \times 10^{6} / \mathrm{ml}\right)$ and followed by the incubation of $50 \mu \mathrm{l}$ of cells for 1 hour at $4^{\circ} \mathrm{C}$ with either $20 \mu \mathrm{l}$ of biotinylated IL-6 or $20 \mu \mathrm{l}$ of biotinylated negative control reagent. 20ul of Avidin-fluorescein was then added to each set of cells and incubation was continued for a further 30 minutes at $4^{\circ} \mathrm{C}$. Cells were then washed two times with the supplied wash buffer, resuspended with $300 \mu \mathrm{l}$ of wash buffer containing $10 \mathrm{ug} / \mathrm{ml}$ propidium iodide (PI) to identify dead cells and examined by flow cytometry. A total of 40000 cells were counted for each analysis. PIpositive/dead cells were subsequently excluded from the IL- 6 binding examination. Fluorescence was measured using the CyAn System with Summit software (Beckman Coulter, Brea, CA).

\section{Cell growth suppression analysis}

DU145, PC-3 or A2058 cells were plated in 96-well plates in a complete culture medium with or without different amount of glucosamine. Two days later, the relative cell numbers per well were determined by the colorimetric Janus green whole-cell stain method. Briefly, culture medium was removed from cell layers, then cells were fixed with $4 \%$ formaldehyde (Thermo Scientific, Rockford, IL; 28906) for $30 \mathrm{~min}$ at room temperature, and followed by vacuum aspiration of the fixative. Subsequently, fixed cell layers were stained with Janus Green Whole-Cell Stain solution (Thermo Scientific, Rockford, IL; 62203) for $5 \mathrm{~min}$ at room temperature. Then the excess stain was removed by vacuum aspiration and cells were washed 4 times with water. The dyes associated with cell layers were then eluted by the addition of $100 \mu \mathrm{l}$ of $0.5 \mathrm{~N} \mathrm{HCl}$ per well and shaking the plates at room temperature for $1 \mathrm{~h}$. The colored dye solutions were read 
in a microplate reader (ASYS UVM340 Microplate Reader, Cambridge UK) at $615 \mathrm{~nm}$. Results were expressed as a percentage of the control without glucosamine. Data were represented as mean +/- standard deviation (SD) from quadruplicate wells. Each cell line was analyzed in three independent experiments.

\section{Competing interests}

The authors do not have any financial or personal relationships with other people or organizations that could inappropriately influence the work described in this manuscript.

\section{Authors' contributions}

VC conceived the idea, designed the study and performed the most of experiments. BG and CS involved in the experiments and manuscript editing. $\mathrm{Kl}$ coordinated and supervised the study and edited the manuscript. All authors have read and approved the final manuscript.

Received: 19 June 2013 Accepted: 13 May 2014

Published: 28 May 2014

\section{References}

1. Chesnokov V, Sun C, Itakura K: Glucosamine suppresses proliferation of human prostate carcinoma DU145 cells though inhibition of STAT3 signaling. Cancer Cell Int 2009, 9:25-36.

2. Levy DE, Darnell JE: STATs: transcriptional control and biological impact. Nat Rev Mol Cell Biol 2002, 3:651-662.

3. Yu H, Jove R: The STATs of cancer - new molecular targets come of age. Nat Rev Cancer 2004, 4:97-105.

4. Mora LB, Buettner R, Seigne J, Diaz J, Ahmad N, Garcia R, Bowman T, Falcone R, Fairclough R, Cantor A, Muro-Cacho C, Livingston S, Karras J, Pow-Sang J, Jove $R$ : Constitutive activation of Stat3 in human prostate tumors and cell lines: direct inhibition of Stat3 signaling induces apoptosis of prostate cancer cells. Cancer Res 2002, 62:6659-6666.

5. Amit-Vazina M, Shishodia S, Harris D, Van Q, Wang M, Weber D, Alexanian R, Talpaz M, Aggarwal BB, Estrov Z: Atiprimod blocks STA3 phosphorylation and induces apoptosis in multiple myeloma cells. Br J Cancer 2005, 93:70-80.

6. Kotha A, Sekharam M, Cilenti L, Siddiquee K, Khaled A, Zervos AS, Carter B, Turkson J, Jove R: Resveratrol inhibits Src and Stat3 signaling and induces the apoptosis of malignant cells containing activated Stat3 protein. Mol Cancer Ther 2006, 5:621-629.

7. Klampfer L: Signal transducers and activators of transcription (STATs): Novel targets of chemopreventive and chemotherapeutic drugs. Curr Cancer Drug Targets 2006, 6:107-121.

8. Okamoto M, Lee C, Oyasu R: Interleukin- 6 as a paracrine and autocrine growth factor in human prostatic carcinoma cells in vitro. Cancer Res 1997, 57:141-146.

9. Heinrich PC, Behrmann I, Muller-Newen G, Schaper F, Graeve L: Interleukin6-type cytokine signaling through the gp130/Jak/STAT pathway. Biochem J 1988, 334:297-314

10. Varki A: Biological roles of oligosaccharides: all of the theories are correct. Glycobiology 1993, 3:97-130.

11. Kornfeld R, Kornfeld S: Assembly of asparagine-linked oligosaccharides. Annu Rev Biochem 1985, 54:631-664.

12. Zhao WY, Takahashi M, Gu JG, Miyoshi E, Matsumoto A, Kitazume S, Taniguchi N: Functional roles of $\mathrm{N}$-glycans in cell signaling and cell adhesion in cancer. Cancer Sci 2008, 99:1304-1310.

13. Moritz RL, Hall NE, Connollly LM, Simpson RJ: Determination of the disulfide structure and $\mathrm{N}$-glycosylation sites of the extracellular domain of the human signal transducer gp130. J Biol Chem 2001, 276:8244-8263.

14. Koch HU, Schwarz RT, Scholtissek C: Glucosamine itself mediates reversible inhibition of protein glycosylation. Eur J Biochem 1979, 94:515-522.

15. McDowell W, Schwarz RT: Dissecting glycoprotein biosynthesis by the use of specific inhibitors. Biochimie 1988, 70:1535-1549.

16. Richard D, Plagemann PGW: Cytochalasin B: inhibition of glucose and glucosamine transport. Proc Natl Acad Sci U S A 1972, 69:1430-1434.

17. Zhen Y, Caprioli RM, Staros JV: Characterization of glycosylation sites of the epidermal growth factor receptor. Biochemistry 2003, 42:5478-5492.
18. Downward J, Parker P, Waterfield MD: Autophosphorylation sites on the epidermal growth factor receptor. Nature 1984, 311(5985):483-485.

19. D'Ambrosio E, Casa B, Bompani R, Scali G, Scali M: Glucosamine sulfate: a controlled clinical investigation in arthrosis. Pharmacotherpeutica 1981, 2:504-508.

20. Quastel JH, Cantero A: Inhibition of tumor growth by D-glucosamine. Nature 1953, 171:252-254.

21. Bekesi JG, Winsler RJ: Inhibitory effects of D-glucosamine on the growth of Walker 256 carcinosarcoma and on protein, RNA, and DNA synthesis. Cancer Res 1970, 30:2905-2912.

22. Tarentino AL, Gomez CM, Plummer TH: Deglycosylation of asparagine-linked glycans by peptide: N-glycosidase F. Biochemistry 1985, 24:4665-4671.

23. Yanagisawa M, Yu RK: N-glycan modulates the activation of gp130 in mouse embryonic neural precursor cells. Biochem Biophys Res Commun 2009, 386:101-104.

24. Contessa JN, Bhojani MS, Freeze HH, Rehemtulla A, Lawrence TS: Inhibition of $\mathrm{N}$-linked glycosylation disrupts receptor tyrosine kinase signaling in tumor cells. Cancer Res 2008, 68:3803-3809.

25. Ling YH, Li T, Perez-Soler R, Haigentz M Jr: Activation of ER stress and inhibition of EGFR N-glycosylation by tunicamycin enhances susceptibility of human non-small cell lung cancer cells to erlotinib. Cancer Chemother Pharmacol 2009, 64:539-548.

26. Shiraishi T, Yoshida T, Nakata S, Noronaka M, Wakada M, Mizutani Y, Miki T, Sakai T: Tunicamycin enhances tumor necrosis factor -related apoptosisinducing ligand-induced apoptosis in human prostate cancer cells. Cancer Res 2005, 65:6364-6370.

27. Bourke CA, Carrigan MJ: Experimental tunicamycin toxicity in cattle, sheep and pigs. Aust Vet J 1993, 70:188-189.

28. Gillies RJ, Robey I, Gatenby RA: Causes and consequences of increased glucose metabolism of cancers. J Nucl Med 2008, 49:24s-42s.

\section{doi:10.1186/1475-2867-14-45}

Cite this article as: Chesnokov et al:: Anti-cancer activity of glucosamine through inhibition of N-linked glycosylation. Cancer Cell International 2014 14:45.

\section{Submit your next manuscript to BioMed Central and take full advantage of:}

- Convenient online submission

- Thorough peer review

- No space constraints or color figure charges

- Immediate publication on acceptance

- Inclusion in PubMed, CAS, Scopus and Google Scholar

- Research which is freely available for redistribution 